\title{
Flux quanta, magnetic field lines, merging - some sub-microscale relations of interest in space plasma physics
}

\author{
R. A. Treumann ${ }^{1,2, *}$, R. Nakamura ${ }^{3}$, and W. Baumjohann ${ }^{3}$ \\ ${ }^{1}$ Department of Geophysics and Environmental Sciences, Munich University, Munich, Germany \\ ${ }^{2}$ Department of Physics and Astronomy, Dartmouth College, Hanover NH 03755, USA \\ ${ }^{3}$ Space Research Institute, Austrian Academy of Sciences, Graz, Austria \\ * visiting: International Space Science Institute, Bern, Switzerland
}

Received: 25 November 2010 - Revised: 23 May 2011 - Accepted: 20 June 2011 - Published: 25 June 2011

\begin{abstract}
We clarify the notion of magnetic field lines in plasma by referring to sub-microscale (quantum mechanical) particle dynamics. It is demonstrated that magnetic field lines in a field of strength $B$ carry single magnetic flux quanta $\Phi_{0}=h / e$. The radius of a field line in the given magnetic field $B$ is calculated. It is shown that such field lines can merge and annihilate only over the length $\ell_{\|}$of their strictly anti-parallel sections, for which case we estimate the power generated. The length $\ell_{\|}$becomes a function of the inclination angle $\theta$ of the two merging magnetic flux tubes (field lines). Merging is possible only in the interval $\frac{1}{2} \pi<\theta \leq \pi$. This provides a sub-microscopic basis for "component reconnection" in classical macro-scale reconnection. We also find that the magnetic diffusion coefficient in plasma appears in quanta $D_{0}^{m}=e \Phi_{0} / m_{\mathrm{e}}=h / m_{\mathrm{e}}$. This lets us conclude that the bulk perpendicular plasma resistivity is limited and cannot be less than $\eta_{0 \perp}=\mu_{0} e \Phi_{0} / m_{\mathrm{e}}=\mu_{0} h / m_{\mathrm{e}} \sim$ $10^{-9} \mathrm{Ohm} \mathrm{m}$. This resistance is an invariant.
\end{abstract}

Keywords. Space plasma physics (Magnetic reconnection)

\section{Introduction}

Central among the concepts of space plasma physics is the concept of magnetic field lines. It had been introduced for practical reasons by Michael Faraday in the 1830s in his ingenious attempt to visualise the direction of magnetic forces in air. In Faraday's view field lines were purely geometrical entities, the vector direction of the magnetic field, not having any physical substance.

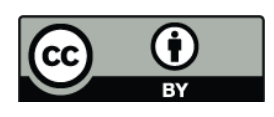

Correspondence to: R. A. Treumann (rudolf.treumann@geophysik.unimuenchen.de)
In space physics the field line concept has been used in a variety of problems, most prominently in the theoretical treatment of magnetic field transport/convection in plasma. Here the magnetic field is considered to be tied to a plasma volume. This convection has been attempted to be described by Euler potentials $\alpha(\mathbf{x}, t), \beta(\mathbf{x}, t)$ (cf., e.g., Stern, 1966, 1967) which are just a different representation of the magnetic vector potential $\mathbf{A}(\mathbf{x}, t)$ under the Coulomb gauge condition $\nabla \cdot \mathbf{A}=0$.

Field lines are also essential in the problem of magnetic reconnection where in addition to convective transport it is asserted that field lines can diffuse, be cut, merge, change topology, and exert stresses on the plasma which cause plasma acceleration and formation of plasma jets (cf., e.g., Paschmann et al., 1979). Though an intuitive picture can be drawn, it still remains unclear what physically is happening when field lines move or merge. In order to arrive at a deeper understanding one should ask for the sub-microscale physics underlying the field-line concept.

In the following we provide a brief inquiry into the physical nature of magnetic field lines. For this one has to recall some simple quantum-mechanical concepts developed long ago $^{1}$ by Landau (1930) and Aharonov and Bohm (1959). This approach, as it turns out, is not entirely academic; it leads to some interesting physical consequences.

\section{Magnetic field lines}

In order to arrive at a deeper understanding of a magnetic field line, one has to acknowledge that the definition of a field line as a physical entity makes sense only in magnetically

\footnotetext{
${ }^{1}$ Landau (1930) treated the quantum-mechanical motion of an electron in a homogeneous magnetic field. Aharonov and Bohm (1959) considered electrons moving around magnetic flux tubes.
}

Published by Copernicus Publications on behalf of the European Geosciences Union. 


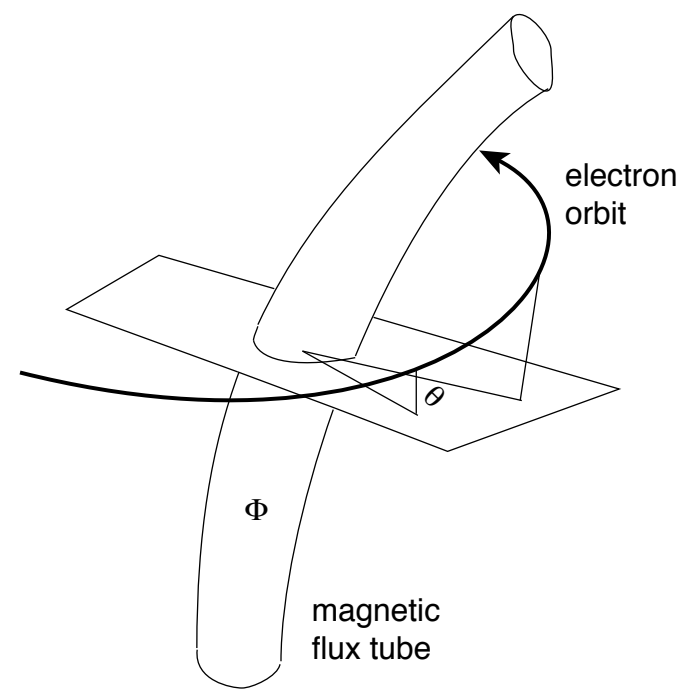

Fig. 1. Geometry of the Aharonov-Bohm effect. The electron (any charged particle) moves at some angle $\theta$ around a magnetic flux tube containing some magnetic flux $\Phi$. Since outside the flux tube the magnetic flux vanishes, the vector potential in this region reduces to a scalar potential which can be gauged away. However, quantum mechanically its effect is remarkable, forcing the flux to be quantised.

active matter, i.e. for charged particles which are sensitive to the presence of magnetic flux, for instance.

\subsection{Field lines carry just one flux quantum}

Under this proposition, a clear definition of a field line can be derived from a quantum-mechanical treatment of magnetic fields in active matter containing electric charges which interact with the magnetic field. Since all charges are attached to masses, one considers the motion of a particle just carrying one elementary charge $e$, e.g. an electron of mass $m_{\mathrm{e}}$. Its motion (which itself is not of interest here) in a magnetic field is described by a Schrödinger equation

$\mathcal{H} \psi(\mathbf{x}, t)=\epsilon \psi(\mathbf{x}, t)$

where $\mathcal{H}$ is the Hamilton operator of the electron, $\psi$ the electron wave function, and $\epsilon$ are the energy eigenvalues of the electron.

There is no need to explicitly solve the Schrödinger equation of motion of an electron (or any other charged particle) in a magnetic field as this was done more than half a century ago by Landau (1930) for an electron moving in a homogeneous magnetic field. The essential result was that the energy of the electron in a magnetic field is quantised

$\epsilon_{\mathrm{L}}\left(p_{\|}, q\right)=\frac{p_{\|}^{2}}{2 m_{\mathrm{e}}}+\hbar \omega_{c e}\left(q+\frac{1}{2}\right) \quad q=0,1,2,3 \ldots$

with quantum number $q$ and parallel momentum $p_{\|}$. Actually, only the perpendicular energy of the gyro-motion is quantised. One easily realises that in a magnetic mirror geometry where the particles oscillate between the mirror points the parallel energy of the electron bounce motion $p_{\|}^{2} / 2 m_{\mathrm{e}} \equiv \epsilon_{\mathrm{b}}=\hbar \omega_{\mathrm{b}}\left(s+\frac{1}{2}\right)$ would also be quantised with bounce frequency $\omega_{\mathrm{b}} \ll \omega_{c e}$ and parallel quantum number $s=0,1,2,3 \ldots q$ thus splitting the Landau energy levels. In fact, under space conditions the lowest Landau energy level is very small; in a magnetic field of $B=100 \mathrm{nT}$ is amounts just to $\epsilon_{\mathrm{L}} \approx 10^{-11} \mathrm{eV}$, which is also the spacing between the Landau levels. Classically these energies form a continuum.

The decisive step towards an understanding of magnetic field lines is done when extending Landau's method and consider the particle motion around one singled-out magnetic flux tube of flux $\Phi$, following Aharonov and Bohm (1959). Again choosing an elementary charge (i.e. an electron) one asks for the electron response to the presence of the flux tube outside the flux tube. In classical physics there is no sign of the magnetic flux contained in the flux tube outside it. This, however, is not true in quantum physics. For outside the flux tube where there are no sources of the magnetic field in the tube, the magnetic vector potential in Schrödinger's equation must be compensated by a gauge field $\phi$, i.e. the gradient of a scalar potential field according to $\mathbf{A}=\nabla \phi$. This introduces a phase factor in the state eigenfunction $\psi(\mathbf{x}, t)$ of the electron, the solution of the Schrödinger equation, in the form

$\psi(\mathbf{x}, t, \phi) \sim \exp \left(-i \frac{e \phi}{\hbar}\right) \psi(\mathbf{x}, t)$

Clearly, this phase factor is unimportant in determining probabilities $|\psi|^{2}$, which is the essence of the gauging, since only probabilities connect to the classical world.

The physical importance of the scalar field $\phi$ becomes clear, however, when returning to the motion of the free electron on some orbit near the magnetic flux tube (cf. Fig. 1). The flux, the electron feels quantum-mechanically is given by the surface integral of the magnetic field $\boldsymbol{B}$ over the part of the field-line cross section $\mathrm{d} \mathbf{F}$, respectively the line integral of the vector potential $\boldsymbol{A}$ along the electron orbit ds

$\Phi=\int_{\text {cross-sect }} \boldsymbol{B} \cdot \mathrm{d} \mathbf{F}=\int_{\text {orbit }} \boldsymbol{A} \cdot \mathrm{d} \mathbf{s}=\int_{\text {orbit }} \nabla \phi \cdot \mathrm{d} \mathbf{s}$

Since the orbit in the scalar potential field $\phi$ can be arbitrarily deformed, one needs to consider only its projection into the plane perpendicular to the flux tube. The value of the line integral then depends only on the electron rotation angle $\theta$ (see Fig. 1), yielding from Eq. (4) for the potential $2 \pi \phi(\theta)=$ $\theta \Phi$. This value, after inserting for $\phi$ into the phase factor, gives just

$\exp \left(-i \theta \frac{e \Phi}{2 \pi \hbar}\right)$

which is the result derived by Aharonov and Bohm (1959). In $\ell$ gyrations the electron would possibly perform around the flux tube, the phase factor would increase by $2 \pi \ell$, and 
the wave function $\psi$ would, artificially, become discontinuous. Since this is unphysical and not permitted in quantum physics, the flux $\Phi=\ell \Phi_{0}$ in the encircled flux tube is a multiple of an elementary flux quantum ${ }^{2}$ (Aharonov and Bohm, 1959)

$\Phi_{0} \equiv 2 \pi \hbar / e \approx 4.1 \times 10^{-15} \mathrm{~Wb}$

(One may notice that this holds for any electric charge $e$ independent on its sign.)

\subsection{Field line radius}

As we will show, the above expression precisely defines a magnetic field line ${ }^{3}$ in magnetically active matter, where the charged particle (electron) is "magnetised", i.e. responds to the presence of the magnetic flux.

The magnetic flux element (quantum) corresponds to a magnetic field of magnitude

$B=\Phi_{0} / \pi \lambda_{m}^{2}$

which, for a given magnetic field strength $B$, defines a (smallest possible) magnetic flux tube radius (Landau, 1930, unaware of the flux quantum, formally arrived from different considerations at a similar "magnetic length", not identifying it with field lines), which is the "radius of a magnetic field line"

$\lambda_{m}=\left(\frac{\Phi_{0}}{\pi B}\right)^{\frac{1}{2}}=\left(\frac{2 \hbar}{e B}\right)^{\frac{1}{2}}$

the narrowest possible flux tube in a magnetic field of given field strength $B$. It just carries one quantum $\Phi_{0}$ of magnetic flux. One may note that $\lambda_{m}$ is independent of the mass of the particle which helped identifying the field line. The particle just served as a carrier of the elementary charge. It depends, however, on the charge $e$ because it is the charge that couples the particle to the field (via the Lorentz force).

This radius is inversely proportional to the square root of the magnetic field $\sqrt{B}$. Strong magnetic fields correspond to narrow field lines, weak magnetic fields have broader field lines. The field line of a weak magnetic field of $B=1 \mathrm{nT}$ has radius of order $\lambda_{m} \sim 10^{-3} \mathrm{~m}$, which is in the detectable meso-scale domain! The weakest magnetic fields in the Universe have strengths of $B \gtrsim 0.1 \mathrm{nT}$. Their field lines have radius $\lambda_{m} \lesssim 1 \mathrm{~cm}$.

Here we have singled out just one field-line fluxtube. Of course, magnetic fluxes may form bundles of such elementary flux quanta, but the smallest possible magnetic flux in

\footnotetext{
${ }^{2}$ This could have been inferred already from simple dimensional reasoning observing that $e \Phi$ has dimension of an action with action quantum $h$.

${ }^{3}$ A first unelaborated hint had already been included by one of us (RT) in a review on reconnection-related problems (Lui et al., 2005).
}

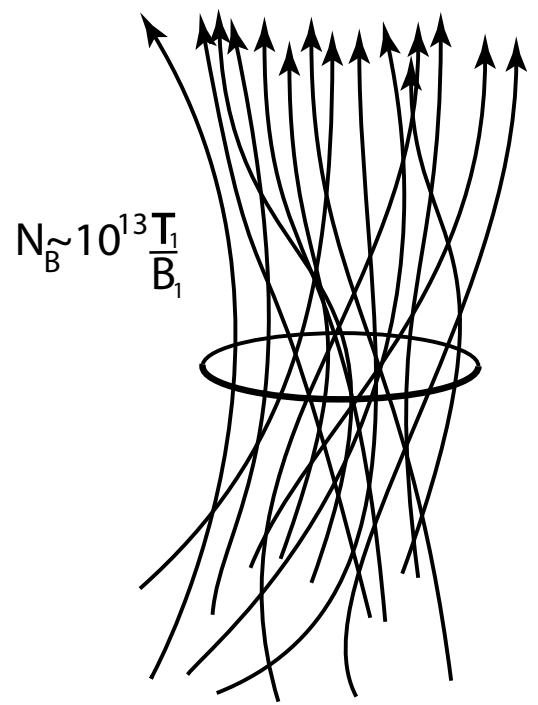

Fig. 2. A few of the $N_{B}=T_{\mathrm{e}} / \hbar \omega_{c e}$ magnetic field lines belonging to the bundle of field lines (flux elements of diameter $2 \lambda_{m}$ ) that are contained in the cross section of an electron gyration $\left(T_{1}=T_{\mathrm{e}}\right.$ in $\mathrm{eV}$, $B_{1}=B$ in $\left.\mathrm{nT}\right)$. Because flux can only annihilate in quanta, implying that only strictly antiparallel field line segments reconnect, none of these field lines can reconnect, however, even though they may get in touch at some inclination angle. Reconnection requires that at least some genuinely antiparallel external field lines are added from the outside. This happens in colliding magnetised plasmas with oppositely directed fields where some field lines will always find their strictly antiparallel partners.

magnetically active matter is given by the ratio $\Phi_{0} \equiv h / e$, the flux carried by a magnetic field line.

This fundamental result of Aharonov and Bohm (1959) yields the precise physical definition of a magnetic field line.

One may calculate the number $N_{B}$ of magnetic field lines contained, for instance, in an electron cyclotron orbit by comparing the area of the electron-gyration circle $\pi r_{c e}^{2}$ with the cross section $\pi \lambda_{m}^{2}$ of a field line. For an electron of temperature $T_{\mathrm{e}}=m v_{\mathrm{e}}^{2} / 2$ in a magnetic field $B$ this yields the large number

$N_{B}=\frac{T_{\mathrm{e}}}{\hbar \omega_{c e}} \approx 10^{13}\left(\frac{T_{\mathrm{e}}}{1 \mathrm{eV}}\right)\left(\frac{B}{1 \mathrm{nT}}\right)^{-1}$

which is the ratio of thermal electron energy to the Landau energy $\hbar \omega_{c e}$ of an electron of cyclotron frequency $\omega_{c e}=$ $e B / m_{\mathrm{e}}$. This number increases with $T_{\mathrm{e}}$ and decreases with magnetic field $\boldsymbol{B}$. In a field of $B=1 \mathrm{nT}$ and for an electron temperature of just $T_{\mathrm{e}}=1 \mathrm{eV}$, this number becomes roughly of the order $N_{B} \sim 10^{13}$. One electron gyration circle thus contains a huge number of magnetic field lines (see Fig. 2). 

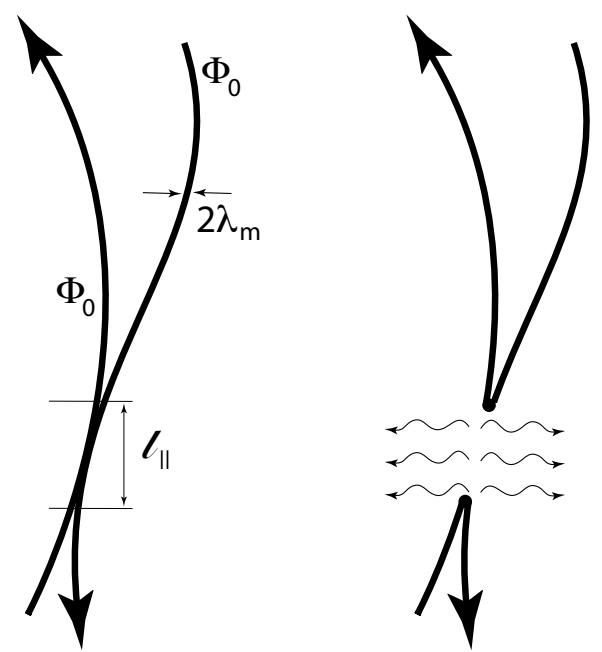

Fig. 3. Annihilation of the strictly antiparallel sections of two contacting magnetic field lines of diameter $2 \lambda_{m}$, each carrying just one flux quantum $\Phi_{0}$. Annihilation proceeds over the antiparallel length $\ell_{\|}$only, thereby creating two new field lines each one, as before, carrying just one flux quantum $\Phi_{0}$. The annihilation releases the magnetic energy stored in the common volume $V_{0}=2 \pi \lambda_{m}^{2} \ell_{\|}$of the two contacting strictly antiparallel flux tube sections. The released energy heats the plasma locally. The strong bending of the remaining (reconnected) parts of the field lines causes relaxation and stretching of the newly reconnected field lines but does not exert any forces on the plasma on the narrow sub-gyroscale of one single field line, causing them to relax as if in vacuum.

\section{3 “Annihilating" field lines}

Asking what, in principle, will be going on when two oppositely directed field lines, i.e. flux elements containing oppositely directed magnetic fluxes, encounter each other, one realises that the outcome of the encounter depends sensitively on the inclination angle under which the two flux elements, respectively field lines, contact. In the following we distinguish between field-line merging and annihilation on the one hand and macroscopic reconnection on the other. The former two apply to the microscopic process when two antiparallel field lines come into contact; the latter is reserved for the macroscopic reconnection effect which involves macroscopic flux tubes and very large numbers of field lines.

\subsection{Merging of two field lines}

Magnetic flux can only be exchanged in quanta $\Phi_{0}=h / e$. Because of this obvious and undeniable property, flux annihilation takes place only when the field lines are precisely anti-parallel a certain distance $\ell_{\|}$along the elementary flux tubes. This is shown schematically in Fig. 3. One should note that because of this reason any obliquely touching field lines (elementary flux tubes) in Fig. 2 cannot annihilate! Moreover, the Lorentz force density between flux tubes is given by $\mathbf{f}_{\text {Lorentz }}=\boldsymbol{j} \times \boldsymbol{B}$. With the current $j=2 B / \mu_{0} \lambda_{m}$ "surrounding" one elementary flux tube this force becomes

$f_{\text {Lorentz }}=\mu_{0}^{-1}\left(2 e B^{4} / \hbar\right)^{\frac{1}{2}}$

For parallel flux tubes (field lines) this force is repulsive keeping the parallel field lines at the largest possible mutual distance, an effect well-known from the tendency of magnetic fields to expand into field-free space, while anti-parallel flux tubes attract each other.

The implication is that, in elementary field-line merging events in which two anti-parallel sections of field lines attract and touch each other, the amount of $2 \Phi_{0}$ of magnetic flux will be annihilated. The annihilated amount of flux is exactly $2 \Phi_{0}$ because each field line carries just one flux quantum, and because flux quanta cannot be divided into half or any fraction such that there is no half flux quantum available which could be added to another half in order to annihilate $1 \Phi_{0}$. Conversely, since field lines are composed of one flux quantum only they contribute $1 \Phi_{0}$ to annihilation in merging, not more and not less. This annihilation happens in a certain time $\Delta t$. Thus the elementary flux annihilation corresponds to the generation of an induced electromotive force (emf)

$|U|=\mathrm{d} \Phi / \mathrm{d} t \approx 2 \Phi_{0} / \Delta t$

Multiplying with the elementary charge, the "equivalent current density" $j$ surrounding the flux element (which is parallel to the emf), and the total volume $V_{0} \approx 2 \pi \lambda_{m}^{2} \ell_{\|}$of the antiparallel sections of the two contacting field lines, we obtain the total power that is generated in such a field-line merging event

$P_{0} \sim \frac{2 B h}{\mu_{0} e} \ell_{\|} \sim 10^{-17}\left(B \ell_{\|}\right) \quad \mathrm{W}$

where $B$ is measured in $\mathrm{nT}$, and $\ell_{\|}$in $\mathrm{m}$. It is assumed this total power is converted into kinetic energy of the plasma. Two annihilating field lines of anti-parallel lengths $\ell_{\|} \sim 1 \mathrm{~m}$ and magnetic field $B \sim 1 \mathrm{nT}$ thus provide a power that is just of the order of $\sim 10^{-17} \mathrm{~W}$.

It is of interest to ask how much time the annihilation of two anti-parallel field lines needs. Previously the corresponding amount of energy $e|U|$ was stored in the volume $V_{0}$ of contacting antiparallel field lines. It thus corresponds to the magnetic energy $V_{0} B^{2} / 2 \mu_{0}$ stored in the two field-line elements such that we find for the annihilation time of two contacting strictly antiparallel flux elements from Eq. (11)

$\Delta t \sim \frac{h}{e|U|} \approx \frac{e \mu_{0}}{B \ell_{\|}} \sim 10^{-17}\left(B \ell_{\|}\right)^{-1} \mathrm{~s}$

where again $B$ is in $\mathrm{nT}$ and $\ell_{\|}$in $\mathrm{m}$. This time is extremely short, i.e. flux elements annihilate instantaneously if and only if they come into antiparallel contact. Microscopically, from the point of view of field lines (or contacting elementary 
flux elements), the question of reconnection thus reduces to two problems: (a) how many flux quanta in an approaching plasma volume element can be turned antiparallel over a certain length $\ell_{\|}$, and (b) how can they be brought into close contact? These questions cannot be answered on the basis of considering the fluid motion of a magnetised plasma volume.

\subsection{Maximum available power}

Nothing is known about the length $\ell_{\|}$of the antiparallel sections of two field lines. Assuming that the field lines would be antiparallel over the ion inertial length $\lambda_{i}$, the largest possible length $\ell_{\|} \simeq \lambda_{i}$ in macroscopic collisionless reconnection (i.e. the total linear extension of the ion-inertial region, see, e.g., Sonnerup, 1979), this number would become the order of $P_{0}\left(\lambda_{i}\right) \sim 2.5 \times 10^{-12}(B \sqrt{N}) \mathrm{W}$, with density $N$ in $\mathrm{cm}^{-3}$. If all the magnetic field lines in one electron gyroradius would reconnect simultaneously over this length we would multiply by the number of field lines $N_{B}$, obtaining (with $T_{\mathrm{e}}$ in $\mathrm{eV}$ )

$P_{0}\left(\lambda_{i}, N_{B}\right) \sim 10\left(T_{e} \sqrt{N}\right) \quad \mathrm{W}$

In the rather extreme case when all the electrons in the ion inertial volume would indeed annihilate their field lines, one had to multiply by the number of electrons $\frac{4 \pi}{3} N \lambda_{i}^{3}$ in the ion inertial volume, and the total power would amount to

$P_{\text {tot }} \sim 10^{9}\left(T_{\mathrm{e}} N^{3}\right) \quad \mathrm{W}$

During a substorm of duration $\Delta t \sim 10^{3} \mathrm{~s}$ this corresponds to a released energy of

$\mathcal{E}_{\text {substorm }} \sim 10^{12} \mathrm{~J}$

some orders of magnitude higher than measured. Clearly, this is an extreme upper limit of what can be obtained in macroscopic reconnection in the magnetospheric tail under the (rather unreasonable) assumption that it is the total number of electrons which (inside the "ion diffusion region" $z<\lambda_{i}$, i.e. in thin current sheets) are responsible for and contribute to reconnection. The energy increases with electron number density $N$ and temperature $T_{\mathrm{e}}$. Apparently, it is independent of the magnetic field $B$, but this is not so because the elementary magnetic flux $\Phi_{0}$ is contained in the fundamental expression Eq. (12).

\subsection{Sub-micro-scale "component reconnection"}

Our claim used so far that the field lines can annihilate only over their antiparallel sections could be challenged on the observation that macroscopic reconnection has been found to depend on the mutual inclination angle of the interacting magnetic fields. In that case one speaks of "component reconnection" referring to the components of the magnetic field involved. In principle something similar applies also to the merging of two field lines with the decisive difference that field lines are very narrow entities of diameter $d=2 \lambda_{m}$.

Nevertheless, assume that two field lines meet under an angle $\theta=\pi$ and $\cos \theta=-1$ over a distance $\ell_{\|}$then the field lines are exactly anti-parallel and once contacting will merge and annihilate their flux elements over the length in question. When the angle is $\theta=\frac{1}{2} \pi$, however, we have $\cos \theta=0$ the field lines are exactly perpendicular, and merging will be inhibited. The field lines in this case touch in a point, and there is no anti-parallel flux component to annihilate. For angles between $\frac{1}{2} \pi<\theta \leq \pi$, on the other hand, a small antiparallel component exists as the antiparallel length shrinks rapidly. The calculation is a little more complicated in this case than in the macro-scale component reconnection case where one assumes that the whole thing happens in an infinitely extended plane. Here, in our case of sub-microscale merging, the two field lines have, however, a transverse extension of only $2 \lambda_{m}$.

During symmetric rotation by an angle $\theta$ around the centre of the anti-parallel contact length $\ell_{\|}$the line of contact shrinks quite fast according to

$$
\ell_{\|}(\theta)=\frac{\lambda_{m} \cos \theta}{\left[\lambda_{m} / \ell_{\|}(\pi)\right] \cos \theta+\sqrt{1-\lambda_{m}^{2} / \ell_{\|}^{2}(\pi)} \sin \theta}
$$

as can easily be shown. When $\theta=\frac{1}{2} \pi$, the length of contact is zero while for $\theta=\pi$ one recovers the full length $\ell_{\|}(\pi) \equiv \ell_{\|}$of contact. Each time the amount of $2 \Phi_{0}$ of flux annihilates in the merging while the available power $P_{0}(\theta)=P_{0} \ell_{\|}(\theta)$ decreases rapidly with angle. So the condition of anti-parallelity is still satisfied but has been modified according to the finite rotation angle $\theta$.

This indeed resembles "component reconnection" but applies here to two single touching field lines thus providing the notion of "component reconnection" a sub-microscopic justification.

\subsection{Field line relaxation after merging}

Another point of discussion refers to the strongly kinked magnetic field line produced in the process of merging and annihilation of flux over the length $\ell_{\|}$. This kink in the magnetic field line is of course not stable. It will relax after merging has ceased and the field line will stretch itself to reach a new equilibrium with minimum curvature. Surely this will happen if only the field line is allowed to stretch. It could thus be claimed that this would be the most important effect on the plasma of the entire merging process similar to that what is known from macroscopic reconnection where the relaxation of the kinked magnetic field lines accelerates the plasma into the famous reconnection jets (first observed by Paschmann et al., 1979) which emanate from the reconnection sites.

However, this is not the case in the sub-microscale merging process. On the sub-microscopic scale the relaxation of the magnetic field lines has no effect at all on the plasma 
as long as the curvature radius, whose initial value is of the order of $\lambda_{m}$, remains much smaller than the electron gyroradius $r_{c e}$. The plasma is affected only when the field has stretched so much that the curvature radius exceeds the electron gyro-radius. Until this happens the electrons remain non-magnetic and the relaxation of the kinked field proceeds in the same way as any magnetic field relaxes in vacuum, i.e. it proceeds at the velocity of light, $c$.

When, after a while, the time-scale of relaxation becomes comparable to the time-scale of plasma oscillations $\Delta t \omega_{\mathrm{e}} \sim 1$ and the curvature radius becomes comparable to the Debyelength, the relaxing fields start feeling the dielectric properties of the plasma; the relaxation then takes place in a medium of dielectric function $\epsilon(\omega)=1-\omega_{e}^{2} / \omega^{2}$ with the effect that in this phase the curvature radius of the relaxing field rapidly increases to reach the electron gyro-radius, while the expansion velocity slows drastically down.

These consequences can be concluded from inspection of the relevant dispersion relation

$k^{2} c^{2}=\omega^{2}-\omega_{\mathrm{e}}^{2}$

For $k c \sim c \lambda_{m}^{-1}$ one has $\omega \sim \Delta t^{-1} \gg \omega_{e}$, and the plasma frequency plays no role; the relaxing field is a high-frequency electromagnetic mode which relaxes with velocity $c$. With increasing curvature one has $k \rightarrow 0$ and $\omega \rightarrow \omega_{e}$ from above, i.e. the relaxation time $\Delta t \sim \omega^{-1}$ increases. Ultimately for $k \sim r_{c e}$ the electrons become magnetised in the field and start feeling its relaxation. Then the above dispersion relation ceases to hold. This has two drastic consequences: firstly, the electron inertia causes a rapid mass loading of the field which retards the further relaxation while, secondly, the relaxing field accelerates the local fraction of electrons up to the electron-Alfvén velocity. Further slow relaxation of the field proceeds until the curvature radius meets the ion gyroradius. Then the expansion of the field is slowed down even further and the ions become accelerated up to the ion-Alfvén velocity generating the famous reconnection jets.

In these last steps, however, many field lines have already become involved and the merging has made the transition from sub-microscopic field-line merging and flux annihilation to reconnection at macroscopic scales. The remaining problem is thus reduced to the understanding of how many a very large number of antiparallel or inclined magnetic field lines, i.e. elementary flux tubes, can be brought into close contact because once they contact each other flux annihilation, magnetic merging, topological change and re-ordering of the magnetic field is inevitable. This is, however, a major problem central to reconnection which lies far outside the scope of this communication.

\section{Magnetic diffusivity}

Before proceeding to the conclusions we briefly note that the existence of a magnetic flux quantum implies as well a small- est quantum $D_{0}^{m}$ of the magnetic diffusivity such that the diffusion coefficient in a magnetic field of flux $\Phi=\ell \Phi_{0}$ can be written as

$D_{\ell}^{m} \sim \ell D_{0}^{m}, \quad \ell \in \mathrm{N}$

This can be realised when multiplying $\Phi_{0}$ by the ratio $e / m_{\mathrm{e}}$ of elementary charge to electron mass yielding

$D_{0}^{m} \sim \frac{e}{m_{\mathrm{e}}} \Phi_{0}=\frac{h}{m_{\mathrm{e}}} \approx 10^{-4} \quad \mathrm{~m}^{2} \mathrm{~s}^{-1}$

which has the correct dimension of a diffusion coefficient: $\left[D^{m}\right]=\mathrm{m}^{2} \mathrm{~s}^{-1}$. Thus, diffusion in a magnetic field proceeds in steps or elementary jumps from field line to field line., which is quite an intuitive picture of the diffusion process.

Correspondingly, the diffusion time $\tau_{D}^{m}$ over a certain length $L$ (measured in meters) is then given by

$\tau_{D}^{m}=10^{4}\left(L^{2} / \ell\right) \quad$ s $\quad \ell=1,2,3 \ldots$

The expression ${ }^{4}$ Eq. (20) can be derived in two ways. First assuming with Bohm that the maximum perpendicular displacement within one electron-gyration time during the elementary diffusion process is just of the order of one field-line diameter

$D^{m} \sim 4 \lambda_{m}^{2} \omega_{c e} / 2 \pi$

This yields immediately for

$D^{m} \simeq \frac{4 \hbar}{\pi m_{\mathrm{e}}}$

Otherwise one may use the definition of the diffusion coefficient through energy

$D^{m} \sim 4 \pi \epsilon_{0 \perp} / m_{\mathrm{e}} \omega_{c e}$

where $\epsilon_{0 \perp}=\frac{1}{2} \hbar \omega_{c e}$ is the perpendicular Landau zero point electron energy (quantum number $q=0$ ). This yields

$D^{m} \simeq \frac{2 \pi \hbar}{m_{e}}$

Both expressions agree with $D_{0}^{m}$ up to a numerical factor of order $O(1)$.

Since otherwise the magnetic diffusivity is defined through resistivity $\eta$, one also has

$D_{0}^{m}=\frac{\eta_{\perp}}{\mu_{0}}$

an equation which immediately shows that the perpendicular ordinary resistivity in a magnetised plasma is itself quantised, $\eta_{\ell \perp}=\ell \eta_{0 \perp}$, and cannot be less than its quantum

$\eta_{0 \perp} \equiv \frac{\mu_{0} e}{m_{\mathrm{e}}} \Phi_{0}=\frac{\mu_{0} h}{m_{\mathrm{e}}} \approx 10^{-9} \quad$ Ohm m

\footnotetext{
${ }^{4}$ Its independence on charge $e$ and magnetic field $\boldsymbol{B}$ identifies it as a general quantum of diffusivity valid for stream lines in a fluid.
} 
This fact restricts the ordinary perpendicular conductivity $\sigma_{\perp}=\sigma_{0 \perp} / \ell$ in a plasma to values

$\sigma_{0 \perp}<10^{9} \quad \mathrm{~S} \mathrm{~m}^{-1}$

Though this upper bound on the plasma conductivity is still high it shows that magnetised plasmas retain a small resistance which is an invariant the origin of which is to be found in the quantum nature of the magnetic flux.

\section{Conclusions}

Summarising, we have clarified the notion of magnetic field lines in plasmas. It turns out that the existence of a magnetic flux quantum also implies the existence in any magnetic field of strength $B$ of a physically narrowest magnetic flux tube, which is a magnetic field line.

Magnetic field lines carry just one flux quantum $\Phi_{0}$, while their radius depends on the local field strength $B$ and shrinks with increasing $B$.

There have been two debatable points in our argument on field-line merging and flux annihilation. The first concerns the question of what strictly antiparallel means. We have clarified this by permitting the flux tubes to be mutually inclined by an angle $\theta$. Two field lines, that is elementary flux tubes carrying one flux quantum $\Phi_{0}$ can merge only when they are strictly anti-parallel over a section of length $\ell_{\|}$along the flux tubes as shown for the ideal case in Fig. 3 . This is required because flux quanta cannot be divided but have to be exchanged in integer numbers. If two bent field lines are mutually inclined while getting into contact at some point in space the projections of one field line onto the other yields a certain length $\ell_{\|}$which, depending on the direction of the field line can be parallel or anti-parallel. This is the length $\ell_{\|}(\theta)$ in question. It involves the angle of inclination $\theta$. Merging and annihilation of flux in integer numbers of quanta becomes possible only when the angle of contact is $\theta>\frac{1}{2} \pi$, implying $\cos \theta<0$. This simple condition yields a precise boundary for the mutually necessary inclination of field lines in order to allow for efficient merging.

Carrying out the calculation of the contact length $\ell_{\|}(\theta)$ confirms that, as required, maximum merging power is obtained at inclination angle $\theta=\pi$, while merging ceases at $\theta=\frac{1}{2} \pi$. The decrease in power with increasing obliquity is drastical because of the narrow width of the contacting field lines. However, though the energy release depends on $\theta$, in each case the constant amount of $2 \Phi_{0}$ in flux will still be annihilated.

The second point concerned the strong kink in the magnetic field which evolved after merging. This kink might lead to suspect that it would be the main ingredient in the merging process in close similarity to reconnection. However, on the sub-microscale the merging takes place in vacuum. This has been discussed at length leading to the insight that the relaxing field lines become mass-loaded and start affecting the plasma when their radius of curvature exceeds the electron gyro-radius.

This takes place in the late evolution of the stretching and relaxation of the field line when many field lines become involved. In this way the sub-microscale physics makes the transition into classical physics at the macro-physical scales.

We have also shown that the existence of flux quanta implies that the diffusivity in a plasma is quantised. The diffusion process can be understood as the particle jumping from field line to field line. This fact implies, in addition, an upper (quantum) bound on the conductivity in a plasma which itself is a constant of nature.

Acknowledgements. This research was part of an occasional Visiting Scientist Programme in 2006/2007 at ISSI, Bern. RT thankfully recognises the assistance of the ISSI librarians, Andrea Fischer and Irmela Schweizer. He highly appreciates the continuous encouragement and support by André Balogh, Director at ISSI, and the discussions with André Balogh and Silvia Perri, all at ISSI, on the present subject. Finally, because of its unconventional approach to the subject of field lines and field line merging, this paper encountered severe problems in passing the referee process. Since it had to be modified several times to satisfy the referees' wishes and to make the presentation clear enough for understanding, the referees (who unfortunately remained anonymous such that they cannot be acknowledged personally) have had substantial participation in the final form of this paper which is unrestrictedly acknowledged at this place.

Topical Editor I. A. Daglis thanks G. Belmont and two other anonymous referees for their help in evaluating this paper.

\section{References}

Aharonov, Y. and Bohm, D.: Significance of electromagnetic potentials in the quantum theory, Phys. Rev., 115, 485-491, 1959.

Landau, L. D.: Diamagnetismus der Metalle, Z. Physik, 64, 629637, doi:10.1007/BF01397213, 1930.

Lui, A. T. Y., Jacquey, C., Lakhina, G. S., Lundin, R., Nagai, T., Phan, T.-D., Pu, Z. Y., Roth, M., Song, Y., Treumann, R. A., Yamauchi, M., and Zelenyi, L. M.: Critical issues on magnetic reconnection in space plasmas, Space Sci. Rev., 116, 497-521, doi:10.1007/s11214-005-1987-6, 2005.

Paschmann, G., Papamastorakis, I., Sckopke, N., Haerendel, G., Sonnerup, B. U. Ö., Bame, S. J., Asbridge, J. R., Gosling, J. T., Russell, C. T., and Elphic, R. C.: Plasma acceleration at the Earth's magnetopause - Evidence for reconnection, Nature, 282, 243-246, doi:10.1038/282243a0, 1979.

Sonnerup, B. U. Ö.: Magnetic field reconnection, in: Solar System Plasma Physics, Vol III, pp. 45-108, edited by: Lanzerotti, L. T., Kennel, C. F., and Parker, E. N., North-Holland, New York, 1979.

Stern, D. P.: The motion of magnetic field lines, Space Sci. Rev., 6, 147-173, doi:10.1007/BF00222592, 1966.

Stern, D. P.: Geomagnetic Euler potentials, J. Geophys. Res., 72, 3995-4005, doi:10.1029/JZ072i015p03995, 1967. 